\title{
Anisotropic Defect-Mediated Melting of Two-Dimensional Colloidal Crystals
}

\author{
C. Eisenmann, U. Gasser, P. Keim, and G. Maret \\ Department of Physics, University of Konstanz, 78457 Konstanz, Germany
}

(Received 2 June 2004; published 3 September 2004)

\begin{abstract}
The melting transition of anisotropic two-dimensional (2D) crystals is studied in a model system of superparamagnetic colloids. The anisotropy of the induced dipole-dipole interaction is varied by tilting the external magnetic field off the normal to the particle plane. By analyzing the time-dependent Lindemann parameter as well as translational and orientational order we observe a 2D smecticlike phase. The Kosterlitz-Thouless-Halperin-Nelson-Young scenario of isotropic melting is modified: dislocation pairs and dislocations appear with different probabilities depending on their orientation with respect to the in-plane field.
\end{abstract}

DOI: 10.1103/PhysRevLett.93.105702

PACS numbers: 64.70.Dv, 61.25.-f, 61.72.Lk, 82.70.Dd

The solid-liquid phase transition is one of the fundamental topics in condensed matter physics. Its study has received new impulses since phase transitions can be observed on a microscopic level by direct imaging in model systems. This is of particular interest for 2D melting, which can be driven by microscopic defects [1-3]. According to the Kosterlitz-Thouless-Halperin-NelsonYoung theory, melting in 2D is mediated by the dissociation of dislocation pairs into free dislocations resulting in a hexatic phase, which still exhibits quasi-long-range orientational order but only short-range translational order. The unbinding of disclinations leads to a second phase transition into the isotropic liquid state. In addition to the importance of the dimensionality of the system, we stress in this letter that the anisotropy of the particle interaction directly influences the orientation of the defects. This leads to a novel phase, and therefore it is also of crucial importance for the nature of the melting transition.

Two dimensional systems with isotropic interaction have been studied intensively in experiments [4-7] as well as in computer simulations of hard disks [8], where the existence of the hexatic phase is still under debate $[9,10]$. However, up to now there have been no experimental results on the influence of an anisotropic interaction on the defects and the melting transition. Theoretical predictions about 2D melting of layers consisting of anisotropic particles have been made by Ostlund and Halperin [11] as well as by Toner and Nelson [12]. Therefore we studied the melting of 2D centered rectangular crystals - which have been predicted theoretically by Froltsov et al. [13] — using a setup of colloidal particles with magnetic dipole-dipole interaction controlled by an external magnetic field. This system is essentially the same as in $[6,7]$ with the additional option to control the anisotropy of the interaction which is achieved by tilting the field away from the direction perpendicular to the particle plane. For small tilting angles up to $20^{\circ}$ we have already reported a two-step melting via a quasihexatic phase similar to melting in the case of isotropic interactions [14]. However, in case of larger tilting angles $\left(23.7^{\circ} \leq \varphi \leq 25.2^{\circ}\right)$ studied now, we find a highly anisotropic centered rectangular crystal lattice, which, close to the melting transition, shows a strong anisotropy in the orientation of defects resulting in melting only along the direction of the in-plane field into a $2 \mathrm{D}$ smecticlike phase.

The experimental system consists of monodisperse polystyrene particles with a diameter of $4.5 \mu \mathrm{m}$ and a mass density of $1.5 \mathrm{~g} / \mathrm{cm}^{3}$ which are confined to the water/air interface of a hanging water droplet by gravity. The particles are superparamagnetic and, therefore, magnetic dipole moments can be induced by applying an external magnetic field $H$. As shown in [15], the dipoledipole interaction dominates all other interparticle interactions, which, as a consequence, need not to be taken into account. The anisotropy of the interaction between the particles is controlled by tilting the magnetic field $\mathbf{H}$ away from the direction vertical to the sample plane by an angle $\varphi$. This leads to a reflection symmetry axis for the pair potential and for the resulting crystal structure that is given by the direction of the in-plane field. While all measurements were carried out at room temperature, the effective system temperature $1 / \Gamma \propto k_{B} T /(\chi H)^{2}$ was controlled by changing the external magnetic field $H$ (see Ref. [14] for an exact definition). $\chi$ is the magnetic susceptibility of a particle. The colloids were observed by digital video microscopy, the size of the field of view was $840 \times 620 \mu \mathrm{m}^{2}$ and contained $\sim 2000$ particles, while the whole sample cell contained roughly 200000 particles. After each change of $\Gamma$ or $\varphi$ the sample was equilibrated for several hours, before particle coordinates were determined and stored for about an hour. Formation of chainlike structures, beginning roughly at tilting angles $\varphi \approx 25^{\circ}$, limits the experimentally accessible $\varphi$ range.

In order to characterize the observed phases, we use the density-density correlation function $g_{\mathrm{G}}\left(\left|\mathbf{r}-\mathbf{r}^{\prime}\right|\right)=$ $\left\langle\exp \left(i \mathbf{G}\left[\mathbf{r}-\mathbf{r}^{\prime}\right]\right)\right\rangle$, the bond-angular correlation function $g_{6}\left(\left|\mathbf{r}-\mathbf{r}^{\prime}\right|\right)=\left\langle\exp \left(i 6\left[\theta(\mathbf{r})-\theta\left(\mathbf{r}^{\prime}\right)\right]\right)\right\rangle$, as well as the projections of the time-dependent Lindemann parameter 


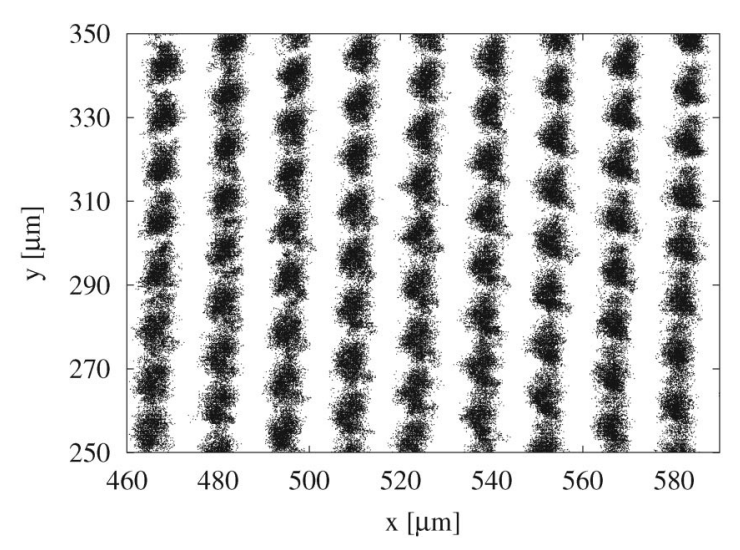

FIG. 1. Particle trajectories during $1 \mathrm{~h}$ of measurement.

$[6,14]$ parallel and perpendicular to the in-plane field, respectively: $\gamma_{\|}(t)=\left\langle\left[\Delta y_{j}(t)-\Delta y_{j+1}(t)\right]^{2}\right\rangle / a^{2}, \gamma_{\perp}(t)=$ $\left\langle\left[\Delta x_{j}(t)-\Delta x_{j+1}(t)\right]^{2}\right\rangle /(a f)^{2}$. G denotes a reciprocal lattice vector, and $\theta(\mathbf{r})$ is the angle between a bond located at $\mathbf{r}$ and the $y$ axis. $j$ and $j+1$ are indices of neighboring particles, $x$ and $y$ are the components of the particle displacement field, $\Delta x(t)=x(t)-x(0)$, and, accordingly, $\Delta y(t)=y(t)-y(0) . a$ is the shorter one of the two lattice constants - which points in the direction of the in-plane component of the magnetic field [14] — and $f$ is the lattice anisotropy determined by Fourier transformation of the particle positions [14]. The in-plane component of the magnetic field is pointing in the $y$ direction. In the case of $g_{6}(r)$, the anisotropy of the lattice influences the orientations of the nearest neighbor bonds. To circumvent this problem, we rescale the particle positions in the $y$ direction in such a way that the anisotropy disappears and the average angle between adjacent bonds in the crystal becomes $60^{\circ}$. Then, the behavior of $g_{6}(r)$ can be compared directly with that of the isotropic case.

The results reported in this Letter were observed for values of the tilting angle $\varphi$ between $23.7^{\circ}$ and $25.2^{\circ}$ and

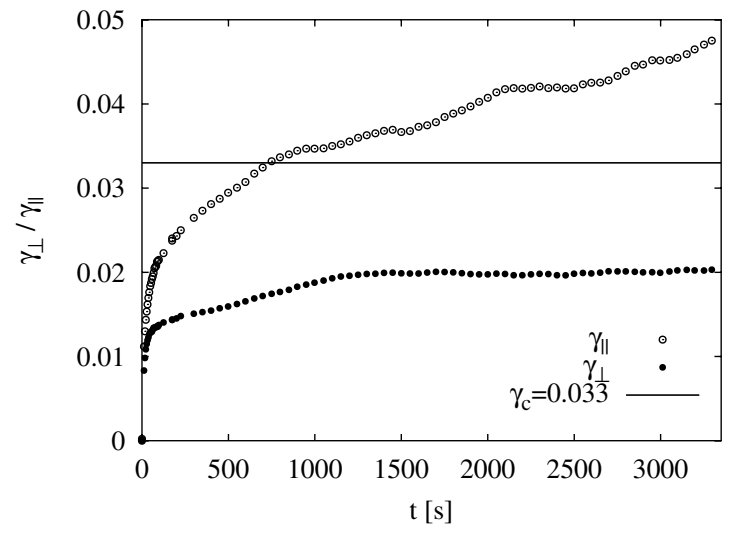

FIG. 2. Time-dependent Lindemann parameter parallel (open circles) and perpendicular (solid circles) to the in-plane field.

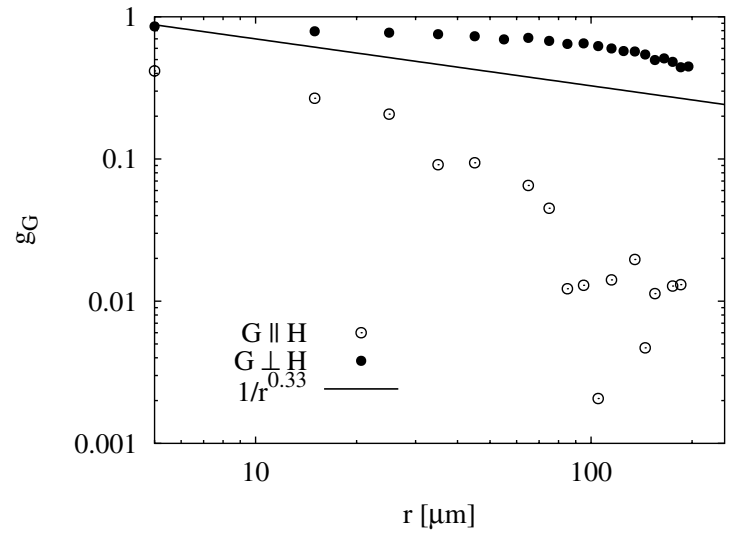

FIG. 3. Density-density correlation function for a reciprocal lattice vector parallel (open circles) and perpendicular (solid circles) to the in-plane field.

for inverse system temperatures $\Gamma$ between 72 and 82 . All the data presented in the figures were observed at a tilting angle $\varphi=24.2^{\circ}$ and an inverse system temperature $\Gamma=$ 76. In the mentioned region we find a phase, which is strongly aligned in columns parallel to the in-plane component of the magnetic field. This is illustrated in Fig. 1, which shows that along the $y$ direction the particles are not confined to a lattice site, whereas perpendicular to the in-plane field the particles are more strongly localized, and, as a consequence, the trajectories are clearly separated from each other. As shown in Fig. 2, the timedependent Lindemann parameter reflects exactly that behavior. $\gamma_{\|}$(open circles) diverges and, therefore, shows liquidlike behavior, while $\gamma_{\perp}$ (solid circles) clearly approaches a constant value at long times as expected for a solid. For comparison the critical value $\gamma_{\mathrm{c}}=0.033$ of the Lindemann parameter, which is observed in the case of isotropic interaction, is shown by the horizontal line. The actual critical value for anisotropic interaction may be different. Moreover, the density-density correlation functions for two reciprocal lattice vectors-one parallel to the in-plane component of $\mathbf{H}$ and the other one perpendicular-show also unlike behavior, as can be seen in Fig. 3. The behavior of the correlation for $\mathbf{G} \perp \mathbf{H}$ is for large $r$ compatible with algebraic decay with an exponent

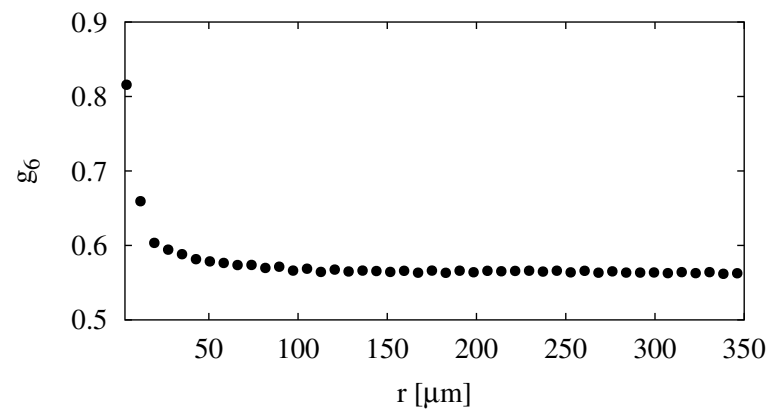

FIG. 4. Bond-angular correlation function. 
smaller than that at melting in a system with isotropic interaction (shown by the line in Fig. 3). However, the decay for $\mathbf{G} \| \mathbf{H}$ is much faster and is fit better by an exponential than an algebraic decay. The orientational order is long range as can be seen in the bond-angular correlation function shown in Fig. 4.

Our data lead to the conclusion that for $\varphi \geq 23.7^{\circ}$ the system melts first parallel to the in-plane component of the field into a smecticlike phase with the particles aligning in columns. These columns are oriented parallel to the in-plane component of the magnetic field leading to long-range orientational order. They are well separated from each other, and quasi-long-range translational order is observed perpendicular to these columns. However, along these columns particles can easily move and their trajectories overlap. Consequently the Lindemann parameter in this direction diverges and translational order is short range parallel to the field.

a

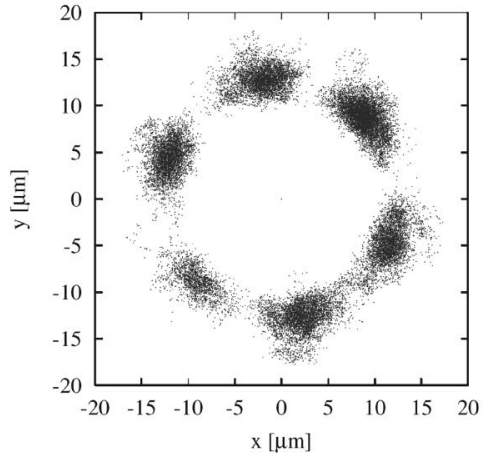

b

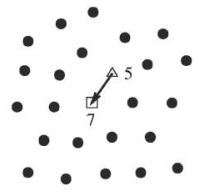

c

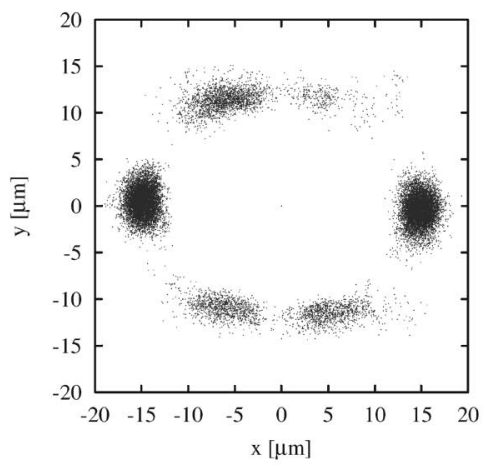

FIG. 5. Orientations (5-7 vectors) of isolated dislocations in measurements with isotropic (a) and anisotropic (c) interaction. Each dot represents the orientation of one dislocation. The orientation of the dislocation was characterized by the vector joining the 5-fold and the 7-fold coordinated particle as shown by the arrow in (b). Particles with a 6-fold coordination are represented by dots.
Since melting in $2 \mathrm{D}$ is closely connected with the dissociation of dislocation pairs (two 5-fold and two 7fold coordinated particles) into free dislocations (a 5-fold and a 7-fold coordinated particle) and disclinations (a single 5-fold or 7-fold coordinated particle), we have studied the differences between the defects in the case of isotropic and anisotropic interaction. For this purpose only isolated defects were taken into account that are defined by having 6 -fold coordinated neighboring particles only. The orientation of a dislocation is given by the vector joining the 5-fold and the 7-fold coordinated particles (5-7 vector) or, equivalently, by its Burgers vector. Both, 5-7 vector and Burgers vector, correspond to one of the six nearest neighbor directions of the triangular lattice. In Fig. 5(a) the 5-7 vectors of the free dislocations in a measurement with isotropic interaction are plotted, and, as expected, a hexagonal pattern is observed. However, when the interaction is anisotropic [Fig. 5(c)], those free dislocations that are oriented perpendicular to the inplane magnetic field (i.e., Burgers vector parallel to the axis of symmetry) are clearly more frequent. This is in agreement with $1 \mathrm{D}$ melting along the direction of the in-

a

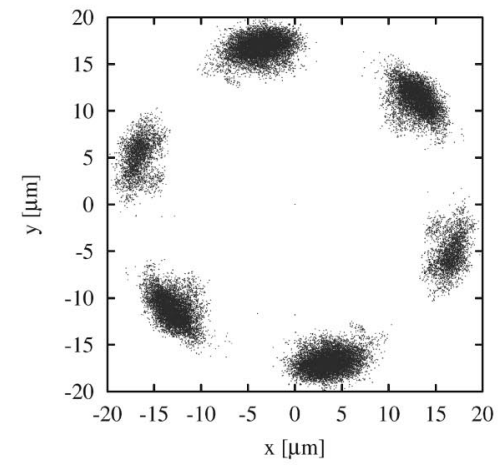

b

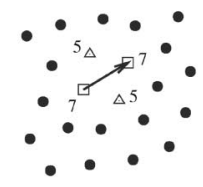

$\mathrm{c}$

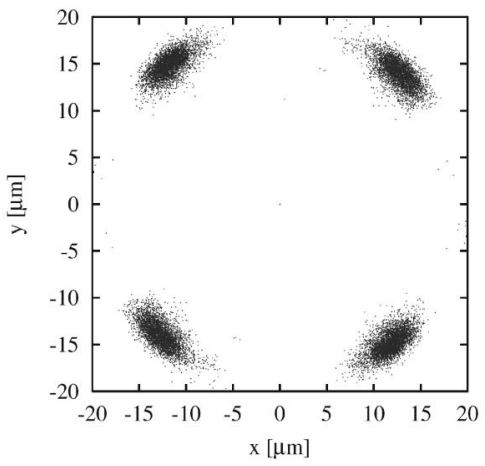

FIG. 6. Analogous to Fig. 5, but for isolated dislocation pairs. As sketched in (b), the orientation of the dislocation pair was characterized by the vector joining the two 7-fold coordinated particles. 
a

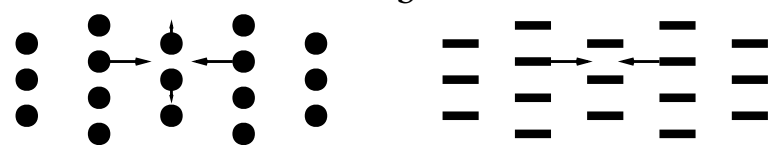

FIG. 7. The movement of particles which is necessary to form the suppressed type of defect is indicated by the arrows for spheres (a) and rodlike particles (b).

plane field, since these dislocations destroy the translational order along this direction. Furthermore, the centered rectangular symmetry of the lattice is visible in Fig. 5(c). The orientation of a dislocation pair is defined by the vector joining the two 7 -fold coordinated particles (7-7 vector). For this type of defect essentially the same effect as for the dislocations is observed, but it is even more pronounced, as can be seen in Fig. 6, where the 7-7 vectors of isolated dislocation pairs from a measurement with isotropic (a) and anisotropic (c) interaction are plotted. In Fig. 6(c) one type of dislocation pair is completely suppressed, and a rectangular pattern is observed as a consequence.

Ostlund and Halperin [11] as well as Toner and Nelson [12] have studied dislocation-mediated melting of anisotropic layers. As there are two types of dislocations in an uniaxial solid, one with the Burgers vector along a reflection symmetry axis (type I) and one lying at angles $\pm \phi_{0}$ from the reflection axis (type II), they suggest that one type of dislocation would unbind first leading in the case of type I melting to a $2 \mathrm{D}$ smectic phase. Although we also find the type I dislocation being favored in our system, we furthermore observe the complete suppression of one type of dislocation pair which has not been predicted in $[11,12]$. This is probably due to the fact that they studied anisotropic particles whereas in our system the particles are isotropic but interact via an anisotropic interaction with a given (external) direction of anisotropy. As sketched in Fig. 7(a), the suppressed type of dislocation pair could form by moving the two particles with long arrows so close together that they become neighbors. However, for a lattice with large enough anisotropy this movement is perturbed by the two particles with short arrows, which would have to be pushed apart. But for rodlike particles this process is not hindered [Fig. 7(b)]. A second discrepancy concerns the orientational order: in case of the anisotropic particles no long-range orientational order has been predicted, whereas for our system the direction of the external field leads to long-range orientational order.

In two independent studies of laser-induced freezing and melting of colloidal systems $[16,17]$ similar structures were observed. However, in that work particles interacting via an isotropic interparticle potential were influenced by an additional external anisotropic (laserlight) potential leading to the reported columnar struc- tures, whereas in our case the interparticle potential itself becomes anisotropic. Nevertheless, the origin of the direction of the anisotropy is external, too.

In conclusion, we have studied melting of anisotropic 2D colloidal crystals consisting of spherical particles interacting via an anisotropic magnetic dipole-dipole interaction. We find a new smecticlike phase, which arises from a phase transition similar to type I melting predicted in [11,12] for anisotropic particles. The resulting phase has long-range orientational order, quasi-longrange translational order perpendicular to the axis of symmetry and short-range translational order along this axis. The time-dependent Lindemann parameter shows crystal-like behavior perpendicular to the symmetry axis and is liquidlike parallel to it. This highly anisotropic character results from an anisotropic orientation of defects: As predicted in [11,12] we find that the type I dislocation with a Burgers vector parallel to the reflection symmetry axis is favored. Beyond these predictions we observe a fourfold symmetry in the orientation of dislocation pairs since one type of dislocation pair is completely suppressed by the anisotropy.

We acknowledge fruitful discussions with D. R. Nelson. This work has been supported by the Deutsche Forschungsgemeinschaft (DFG) by means of subproject C 2 of the SFB Transregio 6 program "Physics of colloidal dispersions in external fields."

[1] J. M. Kosterlitz and D. J. Thouless, J. Phys. C 6, 1181 (1973).

[2] D. R. Nelson and B. I. Halperin, Phys. Rev. B 19, 2457 (1979).

[3] A. P. Young, Phys. Rev. B 19, 1855 (1979).

[4] C. C. Grimes and G. Adams, Phys. Rev. Lett. 42, 795 (1979).

[5] C. A. Murray and D. H. Van Winkle, Phys. Rev. Lett. 58, 1200 (1987)

[6] K. Zahn, R. Lenke, and G. Maret, Phys. Rev. Lett. 82, 2721 (1999).

[7] K. Zahn and G. Maret, Phys. Rev. Lett. 85, 3656 (2000).

[8] For a review, see K. J. Strandburg, Rev. Mod. Phys. 60, 161 (1988).

[9] K. Binder, S. Sengupta, and P. Nielaba, J. Phys. Condens. Matter 14, 2323 (2002).

[10] A. Jaster, cond-mat/0305239.

[11] S. Ostlund and B. I. Halperin, Phys. Rev. B 23, 335 (1981).

[12] J. Toner and D. R. Nelson, Phys. Rev. B 23, 316 (1981).

[13] V. A. Froltsov, R. Blaak, C. N. Likos, and H. Löwen, Phys. Rev. E 68, 061406 (2003).

[14] C. Eisenmann, P. Keim, U. Gasser, and G. Maret, J. Phys. Condens. Matter (to be published).

[15] K. Zahn, J. M. Mendez-Alcaraz and G. Maret, Phys. Rev. Lett. 79, 175 (1997).

[16] A. Chowdhury, B. J. Ackerson, N. A. Clark, Phys. Rev. Lett. 55, 833 (1985).

[17] Q.-H. Wei, C. Bechinger, D. Rudhardt, and P. Leiderer, Phys. Rev. Lett. 81, 2606 (1998). 